\title{
La educación ambiental como herramienta para el cuidado del recurso hídrico
}

\section{Environmental education as a tool for the preservation of water resources}

DOI: http://dx.doi.org/10.17981/cultedusoc.9.3.2018.56

Artículo de investigación. Fecha de recepción: 15/06/2018. Fecha de aceptación: 27/11/2018

Ricardo Romero Paz';

\author{
Jairo Rodríguez Robles; Carlos Rodríguez Rodríguez y Jairo Mendinueta Roca ${ }^{2}$ \\ Institución educativa Departamental San Juan de Palos Prieto (Colombia) \\ institucionsanjuanprieto@gmail.com
}

Para citar este artículo:

Romero, R., Rodríguez, J., Rodríguez, C. y Mendinueta, J. (2018). La educación ambiental como herramienta para el cuidado del recurso hídrico. Cultura. Educación y Sociedad 9(3), 479-484. DOI: http://dx.doi.org/10.17981/cultedusoc.9.3.2018.56

\section{Resumen}

La educación requiere una integración entre comunidad educativa y el ambiente, la práctica pedagógica de las instituciones educativas se nutre de las vinculaciones que establece con los intereses, necesidades y oportunidades que presentan los estudiantes y el entorno. En el corregimiento de San Juan de Palos Prieto, en Pueblo Viejo, la calidad de las fuentes de aguas es inadecuada; razón por la que se planteó un programa de educación ambiental y evaluación de la calidad del agua en la escuela y el corregimiento. A través del trabajo directo con las comunidades se logró identificar los usos, historia y los principales problemas ambientales, destacando el mal uso del recurso, por parte de plantaciones de palma. A través del proyecto se logró la formación y sensibilización de la comunidad en términos ambientales, a fin de mejorar y preservar las fuentes hídricas cercanas a la población, para que estas puedan ser de óptima calidad para los múltiples usos que se le da a nivel doméstico y comunitario.

Palabras clave: Educación ambiental, fuentes hídricas, contaminación.

\begin{abstract}
Education requires an integration between the educational community and the environment, the pedagogical practice of educational institutions is nourished by the links established with the interests, needs and opportunities presented by students and the environment. In the corregimiento of San Juan de Palos Prieto, in Pueblo Viejo, the quality of the water sources is inadequate; This is why an environmental education and water quality evaluation program was set up in the school and the corregimiento. Through direct work with the communities it was possible to identify the uses, history and the main environmental problems, highlighting the misuse of the resource, by palm plantations. Through the project the formation and sensitization of the community in environmental terms was achieved, in order to improve and preserve the water sources close to the population, so that these can be of optimum quality for the multiple uses that are given at the domestic level and community
\end{abstract}

Keywords: Environmental education, water sources, pollution.

1 Líder del grupo de investigación: "Semillero científico de las fuentes".

2 Docentes de la institución educativa Departamental San Juan de Palos Prieto.

- The author; licensee Universidad de la Costa - CUC. 


\section{Introducción}

Actualmente, la educación ambiental va más allá de la educación formal e institucional, para dirigirse a toda la población, con una inclinación no sólo actitudinal, sino también para ampliar la acción a la comunidad, la adquisición de conocimientos y competencias integrales, sociales y de protección al ambiente. Rengifo, Quitiaquez y Mora (2012), destacan la importancia de la educación ambiental y de los docentes que deben liderizar su tarea mediante un trabajo coordinado entre las necesidades de las comunidades y los grupos e instituciones especializadas en la problemática.

La educación ambiental se concibe como un vehículo que favorece la socialización y recontextualización, a partir de los resultados de la investigación científica, tecnológica y social, genera nuevas demandas en conocimientos a los responsables directos de las tareas investigativas básicas; porque esta misión de desarrollar competencias en favor del ambiente debe ser objetivo y campo permanente de la acción investigativa. A través de la exploración y del redescubrimiento del contexto, el individuo entra en contacto con la realidad, se hace creativo e indaga soluciones a sus problemas (Perdomo y Cortez, 2014).

Uno de los tópicos recurrentes en proyectos de educación ambiental es el agua y su cuidado; varios autores han abordado esta problemática desde las escuelas (Díaz, 2000; Flores, 2011; Perdomo y Cortez, 2014), mientras que instituciones como Colciencias (Departamento Administrativo de Ciencia, Tecnología e Investigación) a través del programa Ondas, ha diseñado rutas de investigación específicas para niños y jóvenes con énfasis en el agua (i.e. "Los Navegantes de las Fuentes Hídricas") (Colciencias, 2010).

El corregimiento de San Juan de Palos Prieto, Municipio de Pueblo Viejo, Mag- dalena, es bañado por el caño Roncador, afluente del río Tucurinca, este es una fuente hídrica con un cauce muy leve y superficial en época seca. Este curso de agua recorre un extenso territorio de cultivos de palma de aceite, siendo desviado en algunos tramos para el riego de plantaciones, lo que reduce el volumen del cauce y por consiguiente el uso que puede darle la población para sus actividades básicas diarias. A consecuencia de lo anterior, el abastecimiento de agua del corregimiento es a través de un pozo subterráneo y un tanque elevado, por lo que el servicio de agua en la zona es intermitente, debido a la insuficiencia en el fluido eléctrico.

La Institución Educativa Departamental San Juan de Palos Prieto es una de las instituciones con mayor afectación en términos de salubridad; el restaurante escolar cuenta con pozo subterráneo para el abastecimiento local del recurso, mientras que la calidad del agua de este no puede ser certificada, debido a la presencia de pozas sépticas y las actividades de fumigación en las plantaciones cercanas. Desde la misma escuela surgió el interrogante ¿de qué manera la creación de un programa de educación ambiental llevará al mejoramiento de las fuentes hídricas en San Juan de Palos Prieto?, se planteó la necesidad de evaluar la calidad del agua utilizada para el consumo humano en el corregimiento, sus problemas de contaminación y el nivel de sensibilización y conocimiento de la comunidad sobre sus fuentes de agua.

\section{Metodología}

El estudio se desarrolló bajo un enfoque descriptivo y evaluativo. Mediante la identificación de las prácticas y usos que le dan a los recursos hídricos locales en la comunidad y el plantel educativo, permitiendo además el análisis y la evaluación sobre los conocimientos por parte de la comuni- 
dad (estudiantes, docentes y pobladores cercanos), de la Institución Educativa Departamental San Juan de Palos Prieto, en torno al manejo del recurso, conduciendo desde la práctica docente un apoyo en la enseñanza de la educación ambiental como eje principal en la recuperación de la calidad del agua y el cuidado de los recursos hídricos disponibles.

Como instrumento de recolección de la información se aplicaron la entrevista y la observación directa. Uno de los objetivos principales de estas entrevistas es poder identificar el nivel de conocimiento sobre la importancia de las fuentes hídricas, fuentes de contaminación y usos en San Juan de Palos Prieto. Estas entrevistas fueron diseñadas para dar respuestas abiertas, dándole la posibilidad al entrevistado de argumentar su respuesta (Torrecilla, 2006). Para verificar la información obtenida y el comportamiento de la comunidad educativa frente al uso del agua, se realizaron observaciones directas en campo, dos salidas fueron llevadas a cabo en época de sequía y época de lluvias.

Análisis microbiológico. Para evaluar los agentes contaminantes microbiológicos y el análisis fisicoquímico de los cuerpos de agua, se tomaron muestras en las fuentes de suministro de la comunidad, la institución y del caño Roncador, estas se analizaron con la colaboración del Laboratorio de Calidad de Aguas de la Universidad del Magdalena, que además permitió que los estudiantes investigadores observaran y conocieran el proceso de análisis.

\section{Resultados}

A continuación, se presentan lo hallazgos derivados de los instrumentos aplicados, entrevista estructurada y observación. La entrevista estructurada pasó por varios filtros, entre ellos la redacción y selección de preguntas, hasta la validación de estas.
La entrevista fue aplicada a 15 pobladores de la comunidad de San Juan de Palos Prieto.

Entrevistas. A partir de la información obtenida en las entrevistas realizadas a los pobladores de San Juan de Palos Prieto se determinó que en general la comunidad conoce varios aspectos relacionados con el recurso hídrico de su localidad, tales como su importancia para la vida, las partes que lo componen, el origen y el recorrido de las fuentes hídricas y las amenazas que ponen en peligro la preservación de estas. En este último aspecto, la comunidad identifica a la inadecuada disposición final de residuos, la contaminación, la desforestación, las actividades industriales, el crecimiento de la población y la sobreexplotación del recurso como las principales causas que han conducido al deterioro de las fuentes hídricas.

Como antecedentes importantes, la población argumenta que los desechos agrícolas generados en la producción de palma africana deterioraron las aguas de los caños Roncador y Guamito, siendo hoy en día lugares de drenaje para las fincas, lo que reduce y contamina potencialmente al recurso hídrico y al ambiente. Esta situación ha obligado a la comunidad abastecerse usando otros mecanismos, como el colectar agua de otras fuentes (pozos subterráneos) en tanques y otros recipientes, transportarlo ayudándose de animales de carga. En el caso específico de la Institución Educativa San Juan de Palos Prieto, se abastece de un pozo subterráneo de 12 metros de profundidad construido hace 4 años, al que no se le ha realizado análisis de potabilidad, y suele presentar malos olores, quizá por la presencia de pozas sépticas, poca salubridad y fumigaciones.

Por otra parte, la contaminación del caño Roncador ha incidido sobre la salud de la comunidad, los casos de enfermedades e infecciones estomacales y dérmicas están directamente relacionadas con el 
consumo de agua de esta fuente, por lo que se considera que no es apta para el consumo humano, y solo se utiliza recientemente para actividades agrícolas en el riego de plantaciones, identificándose varias haciendas de la zona como algunos de los sitios en donde se ha desviado el caño para tal fin.

Todos estos factores, han ocasionado disminución de la biodiversidad con la notable desaparición de plantas (la bajagua, tocana y árboles de robles) y de especies animales como aves (garzas, chavarríes y otras aves cantoras), mamíferos (ponche, conejo y guartinaja) y reptiles (hicotea y morrocoyo); además, la perdida de la calidad del recurso, por lo que actividades recreativas y de consumo humano no son posibles como en antaño, para convertirse en lo que es actualmente: un lugar contaminado con poca afluencia de agua, con gran cantidad de maleza y sedimentos en época de sequía, y presencia de desbordamientos para la época de lluvia.

Con la realización de estas entrevistas se logró determinar que según la comunidad la principal causa en el detrimento y desaparición de las fuentes hídricas en San Juan de Palos Prieto es la expansión del cultivo de la palma africana, siendo el cultivo que mayor impacto ocasionó en las fuentes hídricas, considerándose como responsables de esta realidad a los empresarios, agricultores y a la comunidad de dicha localidad. Como medidas de control, los pobladores denuncian ante las autoridades locales la problemática ambiental por la que atraviesa el caño y a su vez se concientiza a toda la comunidad en el cuidado del recurso, enseñando a realizar una disposición adecuada de los desechos sólidos y líquidos. Las variables identificadas pasan a representar agentes inhibidores del desarrollo local de las comunidades, en sus diferentes dimensiones; al respecto, Carrera, Bravo, Marín y Crasto
(2013), señalan la necesidad de coordinar acciones estratégicas que promuevan la integración entre los distintos actores sociales como vía para gestionar procesos de crecimiento y desarrollo.

Observación directa en campo. Se realizaron salidas de campo en época de sequía y lluvias para evaluar y corroborar con los pobladores la percepción que tiene la comunidad sobre el caño Roncador. Durante la sequía se observó un cuerpo de agua con escaso nivel, con muy lento movimiento, casi imperceptible, aguas de color oscuro, basura en cercanías y vegetación escasa. La salida de campo, en época de lluvia permitió un mayor recorrido a lo largo del trayecto del caño Roncador. En los recorridos, se observaron problemas de contaminación y represamiento de sus aguas, sedimentación, basura, y desechos del cultivo de la palma, evidenciado en la coloración del agua, el desvío para riego, a través de canales y motores; además de conocer las especies vegetales y animales habitan en el entorno. A través de esta salida, los niños y niñas pudieron confrontar lo que se manifestó en las entrevistas, con la realidad del caño Roncador.

Respecto a la fuente de agua de la institución educativa, los niños encontraron un pozo a la intemperie y en contacto directo con el medio, cría de animales en patios vecinos, letrinas y depósitos de basuras, como principales focos contaminantes.

Análisis microbiológico. De acuerdo con los análisis de laboratorio, tanto el agua proveniente de las fuentes de agua de la institución educativa como el agua del caño Roncador, usada por la comunidad, se encontró un alto nivel de coliformes totales, muy por encima de los niveles permisibles. También arrojó que son aguas con alta alcalinidad, expresada en carbonato de calcio; contiene $0.4 \%$ de salinidad y alta concentración de sólidos disueltos y en suspensión. 


\section{Discusión}

El proyecto permitió a los estudiantes conocer, a través del contacto con las comunidades, la problemática de las fuentes de agua del corregimiento, fomentando una experiencia formativa desde un enfoque de investigación - acción - participación para la gestión integral del entorno; que además deja de manifiesto que la educación ambiental es una herramienta importante para el intercambio de saberes entre escuelas y comunidades en su área de influencia (Rengifo, Quitiaquez y Mora, 2012).

La experiencia investigativa permitió generar en la población estudiantil un impacto positivo e importante desde la perspectiva de la educación ambiental, el proceso de indagación y apropiación del conocimiento por parte de los actores está mediando entre la población docente y estudiantil; los cuales alcanzaron a caracterizar y analizar la difícil situación de los recursos hídricos naturales que se disponen en la localidad, evidenciando un importante deterioro de la calidad del agua como producto de las malas prácticas administrativas de la política local administrativa, así como un desfavorecimiento por parte del actuar cotidiano de la población. Esto pone de manifiesto la falta de cultura y atención a los recursos causantes del impacto negativo al ambiente, resultando importante que las generaciones actuales y venideras permitan, mediante la educación ambiental, alcanzar una transformación positiva del entorno, en especial desde el cuidado y manejo sostenible del recurso hídrico.

Los resultados del estudio adicionalmente permitieron evidenciar la importancia que tiene el desarrollo del comportamiento proambiental para el desarrollo sostenible, así como la necesidad de fortalecer el desarrollo de políticas y programas educativos, que se orienten a la promoción del cuidado y manejo sostenible y sustentable de los recursos (Cortés, 2011; Crissien-Borrero, 2016; Díaz, 2000)

\section{Referencias}

Colciencias (2010). Los Navegantes de las Fuentes Hidricas. Bogotá, D.C.: Programa Ondas- Colciencias.

Cortés, O. (2011). Comportamiento proambiental y pensamiento económico en la construcción del desarrollo sostenible. Cultura Educación y Sociedad, 2(1), 4356.

Crissien-Borrero, T., Cortés-Peña, O. y Herrera-Mendoza, K. (2016). Pro-Environmental Assessment and Sustainable Consumption of Household Public Services in Barranquilla, Colombia. The European Proceedings of Social \& Behavioural Sciences-EpSBS, 17, 434-440. http://dx.doi.org/10.15405/epsbs.2016.11

Carrera, M., Bravo, O., Marín, F. y Crasto, C. (2013). Futuribles y Futurables para el Desarrollo Sostenible. Lider 22 (15), $39-74$

Díaz, C. (2000). Educación ambiental para el manejo adecuado y racional del recurso hídrico en el colegio san Víctor del Municipio de Supía Caldas. [Tesis de pregrado]. Universidad de Manizales, Manizales.

Orellano, J. (2011). Instituto de Investigaciones Hidráulicas de la UNSJ. Una experiencia de gestión. CETA Centro de Estudios y Tecnología del Agua, 1(1). 74-77. Recuperado de http://www.inv. lab_hidraulica.efn.uncor.edu/wp-content/uploads/2016/04/revista_CETA_1. pdf

Perdomo, A., y Cortez, T. (2014). Diseño de un proceso de educación ambiental para el buen provechamiento de las fuentes hídricas, a través de la resolución de problemas en el contexto escolar [Tesis de pregrado]. Universidad del Valle 
Rengifo, B., Quitiaquez, L. y Mora, F. (2012). La educación ambiental una estrategia pedagógica que contribuye a la solución de la problemática ambiental en Colombia. En, Universidad Nacional de Colombia, XII Coloquio internacional de Geocrítica, Independencias y construcción de estados nacionales: poder, territorialización y socialización, siglos XIX-X. Bogotá, D.C., Colombia. Recuperado de http://www.ub.edu/geocrit/coloquio2012/actas/06-B-Rengifo.pdf
Torrecilla, J. (2006). La entrevista. Madrid: Universidad Autónoma de Madrid. 